\title{
Análise comparativa da estrutura populacional do ermitão endêmico do Atlântico Ocidental Loxopagurus loxochelis (Decapoda, Anomura) em duas regiões do Estado de São Paulo, Brasil
}

\author{
Luciane Ayres-Peres ${ }^{1} \&$ Fernando L. Mantelatto ${ }^{1,2}$
}

\begin{abstract}
1. Laboratório de Bioecologia e Sistemática de Crustáceos, Departamento de Biologia, Faculdade de Filosofia, Ciências e Letras de Ribeirão Preto (FFCLRP), Universidade de São Paulo (USP), Av. Bandeirantes, 3900, 14040-901 Ribeirão Preto, SP, Brasil; Programa de Pós-Graduação em Biologia Comparada, FFCLRP/USP.

2. Autor para correspondência. (flmantel@usp.br)
\end{abstract}

\begin{abstract}
Comparative analysis of population structure of endemic Western Atlantic hermit crab Loxopagurus loxochelis (Decapoda, Anomura) in two regions of state of São Paulo, Brazil. This work characterized the population structure of the hermit crab Loxopagurus loxochelis (Moreira, 1901) in terms of size frequency distribution and sex ratio. Specimens were collected monthly, over a period of one year (from July 2002 to June 2003), in seven transects (from 5 to $35 \mathrm{~m}$ of depth) using fishing boat equipped with two double-rig trawl nets, in Caraguatatuba and Ubatuba regions (state of São Paulo, Brazil). A total of 366 hermit crabs were collected in Caraguatatuba [222 males (60.65\%), 114 non-ovigerous females (31.15\%) and 30 ovigerous females (8.20\%)] and 126 hermit crabs in Ubatuba [81 males (64.28\%), 38 non-ovigerous females (30.16\%) and seven ovigerous females (5.56\%)]. In Caraguatatuba the highest incidence of ovigerous females occurred during winter (July 2002), whereas in Ubatuba, the number was incipient. The cephalothoracic shield length ranged from 2.0 to $7.9 \mathrm{~mm}(5.29 \pm 0.96 \mathrm{~mm})$ in Caraguatatuba, and from 2.7 to $7.5 \mathrm{~mm}(5.32 \pm 0.95 \mathrm{~mm})$ in Ubatuba. The mean size of males was significantly larger than the mean size of females in both regions. Overall sex ratio was in favor of males (1.54:1 in Caraguatatuba and 1.9:1 in Ubatuba). Sexual dimorphism was recorded to L. loxochelis by the presence of males in the largest size classes, following the standard pattern observed in Decapoda. There was an unimodal size distribution for both sexes, with normal distributions in both regions. The higher number of males in relation to females may indicate the existence of different growth and mortality rates between the sexes. Despite of the different geomorphologic characteristics between Caraguatatuba and Ubatuba regions, the dynamics of development was similar for both populations.
\end{abstract}

KEYWORDS. Crustacea, Diogenidae, population, reproduction, sex-ratio.

RESUMO. A estrutura populacional do ermitão Loxopagurus loxochelis (Moreira, 1901) foi analisada por meio da distribuição de freqüência dos animais em classes de tamanho e da razão sexual. Espécimes foram coletados mensalmente no período de um ano (julho de 2002 a junho de 2003), em sete transectos (5 aos $35 \mathrm{~m}$ de profundidade), usando barco equipado com duas redes do tipo double-rig, nas enseadas de Caraguatatuba e de Ubatuba (Estado de São Paulo, Brasil). Em Caraguatatuba foram coletados 366 animais, sendo 222 machos $(60,65 \%), 114$ fêmeas não-ovígeras $(31,15 \%)$ e 30 fêmeas ovígeras $(8,20 \%)$ e, em Ubatuba, 126 ermitões, dos quais 81 machos $(64,28 \%), 38$ fêmeas não-ovígeras $(30,16 \%)$ e sete fêmeas ovígeras $(5,56 \%)$. Em Caraguatatuba, a alta incidência de fêmeas ovígeras ocorreu durante o inverno (julho de 2002), enquanto em Ubatuba, o número foi incipiente. O comprimento do escudo cefalotorácico variou de 2,0 a $7,9 \mathrm{~mm}(5,29 \pm 0,96 \mathrm{~mm})$ em Caraguatatuba, e em Ubatuba, de 2,7 a 7,5 $\mathrm{mm}(5,32 \pm 0,95 \mathrm{~mm})$. A média de tamanho dos machos foi significativamente maior do que das fêmeas em ambas as áreas. A razão sexual total foi favorável aos machos (1,54:1 em Caraguatatuba e 1,9:1 em Ubatuba). Houve maior frequiência de machos nas últimas classes de tamanho, acompanhando os padrões observados em decápodes e evidenciando a existência de dimorfismo sexual em L. loxochelis. A distribuição de freqüência total foi unimodal para ambos os sexos, nas duas áreas. A presença de um número maior de machos em relação a fêmeas pode ser indício de que existam diferentes taxas de crescimento e de mortalidade entre os sexos, além de distribuição espacial diferencial entre estes. Apesar das diferentes características geomorfológicas entre as regiões de Caraguatatuba e Ubatuba, foi observada uma dinâmica de desenvolvimento similar para ambas as populações.

PALAVRAS-CHAVE. Crustacea, Diogenidae, população, reprodução, razão sexual.

Os estudos contínuos e detalhados relacionados à biologia populacional de espécies marinhas com abundância representativa em pequenas áreas como baías e ilhas, constituem uma fonte de informação primordial ao entendimento desse ecossistema (MANTELATTO, 2000). Os crustáceos decápodes compreendem importante parcela da megafauna bentônica deste ambiente. Neste grupo, proporcionalmente a outros componentes, poucos trabalhos têm abordado a estrutura populacional de Anomura, muito provavelmente por se tratar de um grupo cuja maioria dos representantes não apresenta valor comercial, apesar da incontestável relevância e destaque no contexto evolutivo (MANTELATTO \& Sousa, 2000) e na cadeia trófica das comunidades intertidais e de profundidade moderada (Fransozo \& MANTELATTO, 1998). Particularmente entre os ermitões, o conhecimento detalhado sobre os aspectos populacionais de algumas espécies obtido a partir de estudos sistematizados e contínuos está aquém da necessidade ideal para o entendimento da dinâmica de funcionamento desse grupo. Este cenário, fragilizado em relação ao que se conhece sobre outros decápodes, deve-se em parte às dificuldades quanto ao entendimento do intrigante mecanismo que norteia a ocupação e a dependência de conchas de gastrópodes (MANTELATto \& Meireles, 2004; Meireles \& Mantelatto, 2005).

Entre as mais de 800 espécies de ermitões reportadas mundialmente (Ingle, 1993; McLaughlin, 
2003), no Brasil foram, até o momento, registradas 48 espécies de ermitões entre os Diogenidae, Paguridae e Parapaguridae. A família Diogenidae é a mais representativa, com 24 espécies descritas; no Estado de São Paulo são reconhecidas 15 espécies do total de 22 registradas nesse estado (Melo, 1999; Mantelatto et al., 2001; NucCI \& Melo, 2003; NucCI \& Hebling, 2004). Entre estas, Loxopagurus loxochelis (Moreira, 1901), que é endêmica da costa atlântica da América do Sul, distribuindo-se desde a Bahia até a Província de Mar del Plata, na Argentina (Scelzo \& Boschi, 1973; Scelzo, 1976; Melo, 1999), constitui a terceira espécie em abundância e ocorrência no substrato não-consolidado entre profundidades de até $45 \mathrm{~m}$ do litoral paulista (MeIRELES et $a l$. , no prelo).

A biologia dessa espécie vem sendo estudada nas últimas duas décadas com os trabalhos de RIEGER \& D'INCAO (1991), que avaliaram a distribuição de larvas de L. loxochelis na região adjacente à barra de Rio Grande, RS; MARTINELli \& MANTELATTO (1998, 1999), analisaram as populações desta espécie na região norte do litoral paulista (Ubatuba), enfocando a ocorrência de exobiontes nas conchas de gastrópodes e a utilização das mesmas, respectivamente; MantelatTo \& Martinelli (2001) estudaram o crescimento relativo e o dimorfismo sexual; MARTINelli et al. (2002) avaliaram a estrutura populacional e o período reprodutivo em Ubatuba, SP; MANTELATTO $e t$ $a l$. (2004) registraram a distribuição espacial na enseada de Ubatuba, SP; Scelzo et al. (2004) caracterizaram a morfologia dos espermatóforos de duas populações, na Argentina e no Brasil; BERTINI et al. (2004a), enfocaram a distribuição ecológica e o período reprodutivo no litoral norte de São Paulo. Recentemente, BiAgi et al. (2006) realizaram um estudo comparativo da escolha de conchas entre espécimes da Argentina e do Brasil; Mantelatto et al. (2006) avaliaram o status taxonômico desta espécie a partir de análises moleculares, combinado à morfologia e espermiotaxonomia; e ToRati \& Mantelatto (2008) abordaram aspectos sobre o potencial reprodutivo e produção de ovos.

Apesar deste perfil promissor ao entendimento da biologia desta espécie, existe uma lacuna quanto ao conhecimento detalhado dos aspectos inerentes às populações habitantes de áreas até então não exploradas (>17m de profundidade). Neste contexto, caracterizou-se a estrutura populacional do ermitão L. loxochelis em diferentes profundidades nas enseadas de Caraguatatuba e de Ubatuba, litoral norte do Estado de São Paulo, utilizando-se da distribuição de frequiência em classes de tamanho, bem como da razão sexual. De posse dessas informações, averiguou-se a hipótese de que as populações nas duas áreas apresentem padrões de desenvolvimento semelhantes, porém distintos de populações estudadas previamente em áreas mais rasas.

\section{MATERIAL E MÉTODOS}

Área de Estudo. Os espécimes utilizados nesse estudo foram coletados nas enseadas de Caraguatatuba e de Ubatuba, litoral norte do Estado de São Paulo, Brasil. A enseada de Caraguatatuba (23⒌ $51^{\prime} \mathrm{S} ; 4^{\circ} 26^{\prime} \mathrm{W}$ ) abrange uma superfície de $500 \mathrm{~km}^{2}$; encontra-se abrigada da ação direta das ondas e ventos pela ilha de São Sebastião, mostrando ainda uma morfologia de fundo homogênea com suave declividade, acentuando-se somente nas margens de São Sebastião e ao norte do canal (BARROS et al., 1997). A enseada de Ubatuba ( $\left.23^{\circ} 26^{\prime} \mathrm{S} ; 45^{\circ} 02^{\prime} \mathrm{W}\right)$ localiza-se em frente à cidade de Ubatuba e abrange uma área de aproximadamente $16 \mathrm{~km}^{2}$; sua entrada tem aproximadamente $4,5 \mathrm{~km}$, afunilando-se em direção ao continente (Mantelatto \& Fransozo, 1999).

Coleta e análise do material. Os exemplares de $L$. loxochelis foram obtidos mensalmente de julho de 2002 a junho de 2003, no substrato não-consolidado de ambas enseadas. As coletas foram realizadas com um barco de pesca de camarão, equipado com duas redes de arrasto do tipo "double-rig".

Mensalmente foram realizados sete arrastos paralelos ao continente, nas profundidades de $5,10,15$, 20, 25, 30 e 35m, com esforço amostral de 30 minutos em cada arrasto, a uma velocidade de dois nós. Após o término de cada arrasto, as redes foram recolhidas e os animais triados, ensacados, etiquetados e acondicionados em caixas térmicas contendo gelo. Em laboratório, após o descongelamento à temperatura ambiente, os ermitões foram retirados manualmente de suas conchas e, quando necessário, quebrando-as com uma morsa. Os exemplares foram contados e, sob estereomicroscópio óptico, averiguado o sexo considerando-se a posição dos gonóporos. Os animais foram mensurados com paquímetro de precisão $(0,01 \mathrm{~mm})$, anotando-se o Comprimento do Escudo Cefalotorácico (CEC, medido entre a extremidade do rostro até a região mediana da sutura cervical).

A normalidade da distribuição na população em relação a seu tamanho (CEC) foi analisada por meio do teste de Normalidade K-S (Kolmogorov-Smirnov) (ZAR, 1996). O teste Mann-Whitney ( $p<0,05)$ foi aplicado para comparar as médias de comprimento entre machos e fêmeas (ovígeras e não-ovígeras) (ZAR, 1996).

Para a distinção entre indivíduos juvenis e adultos (maduros sexualmente) nas populações estudadas, foram considerados adultos os animais com CEC maior ou igual ao da menor fêmea ovígera coletada para as duas áreas (Garcia \& Mantelatto, 2006), utilizando-se ainda informações sobre o tamanho da maturidade sexual morfológica estabelecido em estudos prévios (Mantelatto \& Martinelli, 2001; Martinelli et al., 2002). As proporções entre machos e fêmeas foram analisadas utilizando-se o teste $\chi^{2}(\mathrm{p}<0,05)\left(\mathrm{ZAR}_{\mathrm{AR}}, 1996\right)$.

Todos os exemplares coletados encontram-se depositados na Coleção de Crustáceos do Departamento de Biologia (CCDB) da FFCLRP/USP, sob os lotes 1485 a 1514 .

\section{RESULTADOS}

$\mathrm{Na}$ enseada de Caraguatatuba foram obtidos 366 espécimes de L. loxochelis durante o estudo. Destes, 205 indivíduos machos $(56,01 \%), 94$ fêmeas não-ovígeras $(25,68 \%), 30$ fêmeas ovígeras $(8,20 \%)$; e 37 juvenis (17 machos e 20 fêmeas) $(10,11 \%)$ (Tab. I). Na enseada de Ubatuba foram coletados 126 espécimes, dos quais 74 machos (58,73\%), 31 fêmeas não-ovígeras (24,60\%) e sete 
fêmeas ovígeras $(5,56 \%)$; os juvenis totalizaram 14 (11,11\%, sendo 7 machos e 7 fêmeas) (Tab. II).

Em Caraguatatuba, houve diferença nas médias de CEC entre machos e fêmeas (tanto as ovígeras quanto as não-ovígeras) $(\mathrm{p}<0,001)$, sendo os machos significativamente maiores que ambas. Já em Ubatuba, foi observada uma diferença significativa do CEC entre machos e fêmeas não-ovígeras $(\mathrm{p}<0,001)$, mas entre machos e fêmeas ovígeras não foi registrada diferença significativa $(p=0,170)$. A menor fêmea ovígera coletada mediu 4,0 mm de CEC, sendo essa medida considerada o limite para a definição de juvenis das populações analisadas.

Os tamanhos médios, máximos e mínimos dos animais amostrados nas duas áreas encontram-se representados na Tab. III. Nas tabelas IV e V estão apresentadas a distribuição dos exemplares nas classes de tamanho em relação às diferentes profundidades de amostragem. Em todas as classes de tamanho, a maior quantidade de ermitões foi obtida nos 20 e $25 \mathrm{~m}$.

A distribuição de freqüência de tamanho (CEC) dos ermitões coletados evidenciou um padrão unimodal e distribuição não-normal em Caraguatatuba $(\mathrm{KS}=0,066$; $\mathrm{p}<0,001)$ e em Ubatuba (KS=0,081; $\mathrm{p}=0,047)$ (Fig. 1).
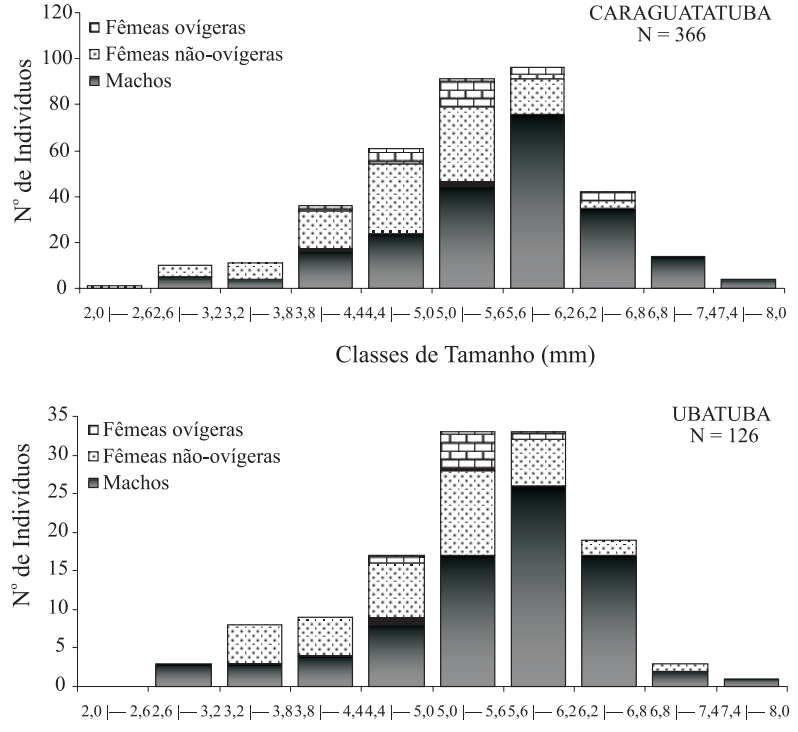

Classes de Tamanho (mm)

Fig. 1. Distribuição de freqüência em classes de tamanho de Loxopagurus loxochelis (Moreira, 1901) para o total de animais coletados mensalmente, de julho/2002 a junho/2003 em Caraguatatuba e Ubatuba, São Paulo.

Tabela I. Número e porcentagem de indivíduos de Loxopagurus loxochelis (Moreira, 1901) coletados mensalmente (de julho/2002 a junho/2003), distribuídos de acordo com os grupos de interesse, em Caraguatatuba, São Paulo (*diferença significativa, p<0,05).

\begin{tabular}{lccccccccccc}
\hline Meses & Machos & $\%$ & $\begin{array}{c}\text { Fêmeas } \\
\text { não-ovígeras }\end{array}$ & $\%$ & $\begin{array}{c}\text { Fêmeas } \\
\text { ovígeras }\end{array}$ & $\begin{array}{c}\text { Juvenis } \\
\text { (machos e fêmeas) }\end{array}$ & Total & $\begin{array}{c}\% \\
\text { Razão } \\
\text { sexual (M/F) }\end{array}$ \\
\hline Julho & 103 & 50,49 & 60 & 29,41 & 16 & 7,84 & 25 & 12,26 & 204 & 100 & $1,3: 1$ \\
Agosto & 04 & 40,00 & 01 & 10,00 & 02 & 20,00 & 03 & 30,00 & 10 & 100 & $1,5: 1$ \\
Setembro & 03 & 37,50 & 03 & 37,50 & & & 02 & 25,00 & 08 & 100 & $0,6: 1$ \\
Outubro & & & 01 & 100 & & & & & 01 & 100 & $0: 1$ \\
Novembro & & & & & & & & & & & \\
Dezembro & 02 & 40,00 & 01 & 20,00 & 01 & 20,00 & 01 & 20,00 & 05 & 100 & $0,6: 1$ \\
Janeiro & 29 & 56,86 & 11 & 21,57 & 06 & 11,77 & 05 & 9,80 & 51 & 100 & $1,55: 1$ \\
Fevereiro & 07 & 77,78 & 02 & 22,22 & & & & & 09 & 100 & $3,5: 1$ \\
Março & 30 & 69,77 & 12 & 27,90 & & & 01 & 2,33 & 43 & 100 & $2,6: 1 *$ \\
Abril & 07 & 53,85 & 02 & 15,38 & 04 & 30,77 & & & 13 & 100 & $1,16: 1$ \\
Maio & 06 & 85,71 & & & 01 & 14,29 & & & 07 & 100 & $6: 1$ \\
Junho & 14 & 93,33 & 01 & 6,67 & & & & & 15 & 100 & $14: 1 *$ \\
\hline Total & 205 & 56,01 & 94 & 25,68 & 30 & 8,20 & 37 & 10,11 & 366 & 100 & $1,54: 1$ \\
\hline
\end{tabular}

Tabela II. Número e porcentagem de indivíduos de Loxopagurus loxochelis (Moreira, 1901) coletados mensalmente (de julho/2002 a junho/2003), distribuídos de acordo com os grupos de interesse, em Ubatuba, São Paulo (*diferença significativa, p<0,05).

\begin{tabular}{|c|c|c|c|c|c|c|c|c|c|c|c|}
\hline Meses & Machos & $\%$ & $\begin{array}{c}\text { Fêmeas } \\
\text { não- ovígeras }\end{array}$ & $\%$ & $\begin{array}{l}\text { Fêmeas } \\
\text { ovígeras }\end{array}$ & $\%$ & $\begin{array}{c}\text { Juvenis } \\
\text { (machos e fêmeas }\end{array}$ & s) & Total & $\%$ & $\begin{array}{c}\text { Razão } \\
\text { sexual (M/F) }\end{array}$ \\
\hline Julho & 14 & 77,78 & 01 & 5,56 & & & 03 & 16,66 & 18 & 100 & $5: 1 *$ \\
\hline Agosto & 01 & 100 & & & & & & & 01 & 100 & $1: 0$ \\
\hline \multicolumn{12}{|l|}{ Setembro } \\
\hline Outubro & 02 & 66,67 & & & & & 01 & 33,33 & 03 & 100 & $2: 1$ \\
\hline Novembro & 06 & 50,00 & 03 & 25,00 & & & 03 & 25,00 & 12 & 100 & $2: 1$ \\
\hline Dezembro & 05 & 41,67 & 03 & 25,00 & 01 & 8,33 & 03 & 25,00 & 12 & 100 & $1: 1$ \\
\hline Janeiro & 20 & 50,00 & 14 & 35,00 & 03 & 7,50 & 03 & 7,50 & 40 & 100 & $1,4: 1$ \\
\hline Fevereiro & 02 & 66,67 & & & & & 01 & 33,33 & 03 & 100 & $3: 0$ \\
\hline Março & 04 & 66,67 & 02 & 33,33 & & & & & 06 & 100 & $2: 1$ \\
\hline Abril & 01 & 33,33 & 02 & 66,67 & & & & & 03 & 100 & $0,5: 1$ \\
\hline Maio & 04 & 44,44 & 04 & 44,44 & 01 & 11,12 & & & 09 & 100 & $0,8: 1$ \\
\hline Junho & 15 & 78,94 & 02 & 10,53 & 02 & 10,53 & & & 19 & 100 & $3,75: 1 *$ \\
\hline Total & 74 & 58,73 & 31 & 24,60 & 07 & 5,56 & 14 & 11,11 & 126 & 100 & $1,9: 1$ \\
\hline
\end{tabular}


Tabela III. Comprimento do escudo cefalotorácico (CEC-mm) dos indivíduos de Loxopagurus loxochelis (Moreira, 1901) coletados mensalmente (de julho/2002 a junho/2003), distribuídos de acordo com os grupos de interesse, em Caraguatatuba (CAR) e Ubatuba (UBA), São Paulo.

\begin{tabular}{|c|c|c|c|c|c|c|c|c|}
\hline \multirow[t]{2}{*}{ Grupos de Interesse } & \multicolumn{2}{|c|}{ CEC (mínimo) } & \multicolumn{2}{|c|}{ CEC (máximo) } & \multicolumn{2}{|c|}{ CEC (média \pm desvio padrão) } & \multicolumn{2}{|c|}{ Total de animais } \\
\hline & CAR & UBA & CAR & UBA & CAR & UBA & CAR & UBA \\
\hline Machos & 4,0 & 4,1 & 7,9 & 7,5 & $5,71 \pm 0,74$ & $5,67 \pm 0,71$ & 205 & 74 \\
\hline Machos jovens & 2,6 & 2,7 & 3,9 & 3,8 & $3,46 \pm 0,43$ & $3,21 \pm 0,43$ & 17 & 07 \\
\hline Fêmeas não-ovígeras & 4,1 & 4,0 & 6,7 & 7,1 & $5,08 \pm 0,56$ & $5,20 \pm 0,68$ & 94 & 31 \\
\hline Fêmeas jovens & 2,0 & 3,3 & 3,9 & 3,9 & $3,40 \pm 0,49$ & $3,60 \pm 0,24$ & 20 & 07 \\
\hline Fêmeas ovígeras & 4,0 & 4,7 & 6,5 & 6,1 & $5,26 \pm 0,64$ & $5,27 \pm 0,44$ & 30 & 07 \\
\hline Total de animais & 2,0 & 2,7 & 7,9 & 7,5 & $5,29 \pm 0,96$ & $5,32 \pm 0,95$ & 366 & 126 \\
\hline
\end{tabular}

Tabela IV. Distribuição dos indivíduos de Loxopagurus loxochelis (Moreira, 1901) coletados de julho/2002 a junho/2003 em função das classes de tamanho (comprimento do escudo cefalotorácico) em relação às profundidades em Caraguatatuba, São Paulo ( $\sigma^{\prime}$, machos, ${ }^{\prime}$ fêmeas não-ovígeras; +, fêmeas ovígeras; *, presença de fêmea ovígera; nenhum exemplar foi capturado nos $35 \mathrm{~m})$.

\begin{tabular}{|c|c|c|c|c|c|c|c|}
\hline \multirow[t]{2}{*}{ Classes de tamanho (mm) } & \multicolumn{6}{|c|}{ Profundidades $^{\dagger}(\mathrm{m})$} & \multirow{2}{*}{$\begin{array}{c}\text { Proporção } \\
\text { ơ: }+ \text { : } 90\end{array}$} \\
\hline & 05 & 10 & 15 & 20 & 25 & 30 & \\
\hline $2,0 \mid-2,6$ & 0 & 0 & 0 & 1 & 0 & 0 & $0: 1: 0$ \\
\hline $2,6 \mid-3,2$ & 1 & 2 & 0 & 6 & 0 & 1 & $5: 5: 0$ \\
\hline $3,2 \mid-3,8$ & 0 & 0 & 0 & 8 & 1 & 2 & $4: 7: 0$ \\
\hline $3,8 \mid-4,4$ & 2 & 1 & 2 & $24 *$ & 6 & 1 & $16: 18: 2$ \\
\hline $4,4 \mid-5,0$ & $2 *$ & 7 & $6 *$ & $33 *$ & $12 *$ & 1 & $24: 30: 7$ \\
\hline $5,0 \mid-5,6$ & 5 & $7 *$ & $3 *$ & $57 *$ & $18 *$ & 1 & $44: 35: 12$ \\
\hline $5,6 \mid-6,2$ & $7 *$ & $11 *$ & 6 & $50 *$ & 21 & 1 & $76: 15: 5$ \\
\hline $6,2 \mid-6,8$ & 3 & 2 & 2 & $28 *$ & 7 & 0 & $35: 3: 4$ \\
\hline $6,8 \mid-7,4$ & 1 & 3 & 0 & 5 & 5 & 0 & $14: 0: 0$ \\
\hline $7,4 \mid-8,0$ & 2 & 0 & 0 & 0 & 2 & 0 & $4: 0: 0$ \\
\hline
\end{tabular}

Tabela V. Distribuição dos indivíduos de Loxopagurus loxochelis (Moreira, 1901) coletados de julho/2002 a junho/2003 em função das classes de tamanho (comprimento do escudo cefalotorácico) em relação às profundidades em Ubatuba, São Paulo ( $\sigma^{\lambda}$, machos, + , fêmeas não-ovígeras; ơ, fêmeas ovígeras; *, presença de fêmea ovígera, ${ }^{\dagger}$, nenhum exemplar foi capturado nos $0,5,30$ e $35 \mathrm{~m}$ ).

\begin{tabular}{|c|c|c|c|c|c|}
\hline \multirow{2}{*}{$\begin{array}{l}\text { Classes de } \\
\text { tamanho (mm) }\end{array}$} & \multicolumn{4}{|c|}{ Profundidades $^{\dagger}(\mathrm{m})$} & \multirow{2}{*}{$\begin{array}{c}\text { Proporção } \\
\text { O: : P: PO+ }\end{array}$} \\
\hline & 10 & 15 & 20 & 25 & \\
\hline $2,0 \mid-2,6$ & 0 & 0 & 0 & 0 & 0 \\
\hline $2,6 \mid-3,2$ & 0 & 0 & 1 & 2 & $3: 0: 0$ \\
\hline $3,2 \mid-3,8$ & 0 & 0 & 2 & 6 & $3: 5: 0$ \\
\hline $3,8 \mid-4,4$ & 0 & 0 & 4 & 5 & $4: 5: 0$ \\
\hline $4,4 \mid-5,0$ & 1 & 1 & $12 *$ & 3 & $8: 8: 1$ \\
\hline $5,0 \mid-5,6$ & 0 & 2 & $27 *$ & 4 & $17: 11: 5$ \\
\hline $5,6 \mid-6,2$ & 1 & 1 & $29 *$ & 2 & $26: 6: 1$ \\
\hline $6,21-6,8$ & 1 & 0 & 16 & 2 & $17: 2: 0$ \\
\hline $6,8 \mid-7,4$ & 0 & 0 & 3 & 0 & $2: 1: 0$ \\
\hline $7,4 \mid-8,0$ & 0 & 0 & 1 & 0 & $1: 0: 0$ \\
\hline
\end{tabular}

As populações apresentaram unimodalidade na distribuição de freqüência sazonal para todos os grupos analisados (machos, fêmeas não-ovígeras e ovígeras). Na primavera, houve uma tendência à bimodalidade em ambas enseadas.

A razão sexual total mostrou-se significativamente favorável para os machos $\left(1,54: 1 ; \chi^{2}=34,71 ; \mathrm{p}<0,05 \mathrm{em}\right.$ Caraguatatuba e $1,9: 1 ; \chi^{2}=11,83 ; \mathrm{p}<0,05$, em Ubatuba). Observou-se que, em relação à frequiência dos indivíduos por classe de tamanho, à medida que o tamanho dos animais aumenta, a razão sexual favorece os machos, verificando-se que nas primeiras classes a razão foi próxima de 1:1 (Fig. 2).

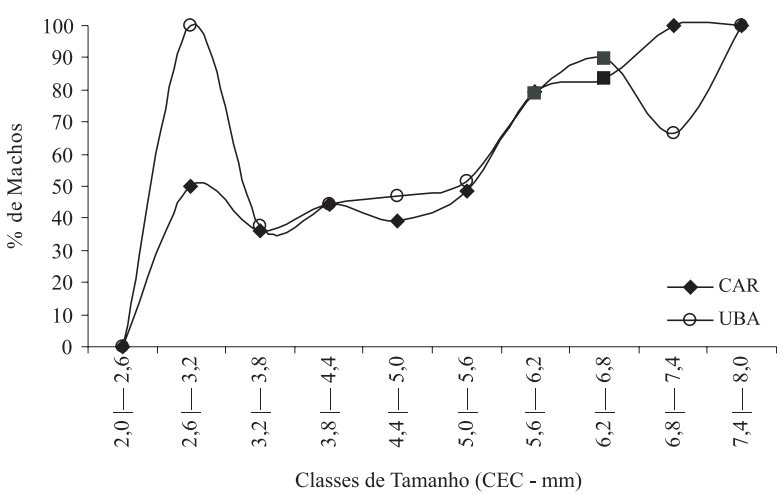

Fig. 2. Porcentagem de machos de Loxopagurus loxochelis (Moreira, 1901) em relação ao total de indivíduos coletados nas classes de tamanho analisadas (CEC-mm) $(\mathbf{\square}=$ diferença significativa, $\mathrm{p}<0,05)$. Indivíduos coletados de julho/2002 a junho/2003 em Caraguatatuba (CAR) e Ubatuba (UBA), São Paulo.

\section{DISCUSSÃO}

O estudo sobre L. loxochelis no litoral norte paulista, revelou ampla dominância em número de indivíduos na enseada de Caraguatatuba em relação à Ubatuba. Tal fato deve-se, possivelmente, às características geomorfológicas de Caraguatatuba. Caraguatatuba encontra-se sujeita a um hidrodinamismo mais homogêneo devido à presença de obstáculos físicos representados pelas ilhas Vitória, Búzios e principalmente pela ilha de São Sebastião, refletindo-se na formação de uma área de deposição de sedimentos finos, 
especialmente na fração silte (PIRES-VANIN et al., 1993). Ubatuba é uma área com maior impacto de correntes marítimas, representadas principalmente por três massas de água: Água Central do Atlântico Sul (ACAS), Água Costeira (AC) e Água Tropical (AT) (CASTRO-Filho et al., 1987), que atingem também a região de Caraguatatuba, porém com diferente intensidade. Esta influência tem sido observada em populações de outros decápodes estudados nesta região, incluindo ermitões (FRANSOZO et al., 1998; MANTELATTO \& Garcia, 2002), camarões (Costa et al., 2000) e caranguejos braquiúros (Bertini \& FRANSOZO, 2004; BERTINI et al., 2004b).

Foi caracterizado um dimorfismo sexual para a espécie em estudo, com machos atingindo maior tamanho do que as fêmeas em ambas as áreas de estudo. Esse padrão é característico em crustáceos decápodes, sendo o mesmo já registrado para $L$. loxochelis por MARTINELLI et al. (2002) e BERTINI et al. (2004a), bem como para outras espécies de ermitões no litoral paulista [e.g. Pagurus brevidactylus (Stimpson, 1858) (NEgReIROS-Fransozo et al., 1991; MANTELATTO et al., 2005); Paguristes tortugae Schmitt, 1933 (Negreiros-Fransozo \& Fransozo, 1992; Mantelatto \& Sousa, 2000); Clibanarius vittatus (Bosc, 1802) (Negreiros-Fransozo et al., 1991; ReIgada \& SANTOS, 1997); Calcinus tibicen (Herbst, 1791) (FRANSOZO \& Mantelatto, 1998); Clibanarius antillensis (Stimpson, 1859) (Negreiros-Fransozo et al., 1991; Turra \& Leite, 1999); Petrochirus diogenes (Linnaeus, 1758) (BERTINI \& Fransozo, 2000) e Paguristes erythrops Holthuis, 1959 (Garcia \& Mantelatto, 2001)].

Segundo AbRAms (1988), três fatores parecem ser os prováveis responsáveis pelo dimorfismo que ocorre em ermitões, estendido aqui para L. loxochelis. O primeiro deles é o direcionamento diferencial de energia para o crescimento. O segundo é a seleção sexual, onde machos maiores apresentam maior sucesso na obtenção de fêmeas para o acasalamento. E o terceiro: o dimorfismo sexual pode propiciar prioridade na escolha da concha mais adequada. No estudo de AsakuRA (1987) com Diogenes nitidimanus Terao, 1913, aquele autor relatou que os fatores mais importantes na hierarquia competitiva dos sistemas de acasalamento dos ermitões são o tamanho do corpo e da quela, respectivamente. Em experimentos de laboratório, machos maiores sempre venceram a competição por fêmeas maduras, corroborando a hipótese da seleção sexual de ABrams (1988). Nas populações de L. loxochelis houve maior proporção de machos em relação às fêmeas, sendo possível que o maior tamanho atingido por esses animais represente vantagem seletiva durante a competição pelo reduzido número de fêmeas, bem como no mecanismo de seleção e ocupação de conchas (BIAGI et al., 2006).

Os menores indivíduos coletados nas enseadas de Caraguatatuba e Ubatuba apresentaram tamanhos (CEC) muito próximos ao observado por MARTINELLI et al. (2002) em Ubatuba (2,5 mm). Mantelatto \& Martinelli (2001) sugeriram, a partir da análise do crescimento relativo e da menor fêmea ovígera coletada, que a maturidade sexual em $L$. loxochelis ocorreria entre 4,5 e 6,0 mm de CEC. No presente estudo, obteve-se um tamanho menor em Caraguatatuba, sugerindo que a maturidade morfológica pode ocorrer em dimensões menores de CEC (embora não tenham sido realizadas análises quanto à maturidade gonadal e fisiológica dos animais). Já BERTINI et al. (2004a) observaram que fisiologicamente a maturidade sexual de L. loxochelis inicia-se próximo aos 3,5 mm de CEC. Tais diferenças encontradas entre estes estudos, muito provavelmente devem-se à variabilidade intra-específica com relação ao tamanho, bem como a eficiência nas metodologias de amostragem em diferentes áreas que podem abrigar parcelas diferentes da população e.g. maior número de juvenis e fêmeas ovígeras.

Fêmeas ovígeras ocorreram em maior número no inverno em Caraguatatuba. $\mathrm{Na}$ enseada de Ubatuba um número incipiente de fêmeas ovígeras foi coletado, corroborando os resultados de MARTINelli et al. (2002) de que a enseada de Ubatuba não é um local propício à reprodução. Tais fêmeas podem estar fora da enseada (áreas offshore), com maiores profundidades e temperaturas menores, condições propícias ao desenvolvimento desta espécie predominante de águas frias (MANTelatto et al., 2004). Bertini et al. (2004a) verificaram que as fêmeas ovígeras, embora com ocorrência anual, apresentaram pico reprodutivo no inverno (64,4\% das fêmeas ovígeras coletadas).

Possivelmente, a ausência de fêmeas ovígeras no período da primavera, em Caraguatatuba, tenha ocorrido devido a uma baixa captura desses indivíduos em função do período e da amostragem. O baixo número de fêmeas ovígeras acompanhou o baixo número de ermitões capturados durante a primavera, semelhante ao observado por Meireles et al. (2006). Aqueles autores ao estudar uma população de Pagurus exilis (Benedict, 1892) na mesma localidade e durante o período do presente estudo coletaram apenas 29 indivíduos (i.e. 3,77\% da população de $P$. exilis foram coletados durante a primavera).

A captura reduzida desses animais, muito provavelmente, tenha ocorrido como resposta a uma pressão seletiva (abiótica ou biótica) que atingiu ambas as populações, induzindo a uma migração dos animais para outras áreas, sendo que tanto $L$. loxochelis como $P$. exilis (Meireles et al., 2006) nas demais estações apresentaram um número expressivamente maior de indivíduos capturados em relação à primavera. Esta condição é corroborada por outros estudos (FRANSOZO et al., 1998 e Martinelli et al., 2002 na região de Ubatuba), que também demonstraram maior ocorrência de indivíduos durante as demais estações, excetuando-se o inverno, quando a população de $L$. loxochelis apresenta maior proporção de indivíduos.

Em Ubatuba, MarTinelli et al. (2002) inferiram que o baixo número de fêmeas ovígeras de L. loxochelis coletadas naquela enseada poderia estar relacionado ao fato de que as mesmas utilizam os recursos da enseada (conchas, sedimento, alimento) para o crescimento e não somente para a reprodução e, portanto, estariam dirigindose para outras áreas (mais profundas) para fazê-lo. Porém, naquele estudo as coletas estenderam-se apenas até $20 \mathrm{~m}$, enquanto que no presente trabalho as amostragens foram realizadas até $35 \mathrm{~m}$ e as fêmeas ovígeras não foram coletadas em regiões mais profundas do que no estudo supracitado. Há fortes indícios de que as fêmeas ovígeras prefiram locais com baixas temperaturas, sendo sua 
presença mais relacionada a esse fato do que propriamente a maiores profundidades. Entretanto, RIEGER \& D' INCAO (1991) reportaram a presença de juvenis e adultos de $L$. loxochelis em áreas litorâneas a profundidades de até $50 \mathrm{~m}$ na Barra de Rio Grande/RS, região com temperaturas médias inferiores às registradas para o litoral paulista.

Também foi observada baixa freqüência de fêmeas ovígeras em Mar del Plata, Argentina (M. A. Scelzo com. pes.), onde apenas $7 \%$ da população de L. loxochelis (de 453 exemplares) apresentou-se nesta condição, com pico de ocorrência registrado nos meses de verão. O baixo número de fêmeas ovígeras corrobora a existência de um eficiente desenvolvimento embrionário e alto potencial reprodutivo. De acordo com recente estudo, há fortes indícios que esta espécie de ermitão possui a capacidade de copular várias vezes, intensificando o esforço reprodutivo (TORATI \& MANTELATTO, 2008) e maximizando o sucesso das larvas. Este contexto explicaria a constante ocorrência de L. loxochelis na região de Ubatuba (Fransozo et al., 1998).

Geralmente, crustáceos de áreas temperadas apresentam reprodução sazonal com pico nos meses quentes, enquanto que em animais de áreas tropicais e subtropicais a reprodução é normalmente contínua ao longo do ano, mas geralmente concentrada nos meses de verão (ASAKURA \& KIKUCHI, 1984). Todavia, L. loxochelis apresentou um modelo diferenciado com reprodução contínua ao longo do ano, porém, com pico sazonal concentrado no inverno (julho de 2002), embora esse representado por um número pouco expressivo de fêmeas ovígeras. Esse resultado está de acordo com o observado por LANCASTER (1988) para Pagurus bernhardus (Linnaeus, 1758) na Inglaterra e também por BERTINI et al. (2004a) para L. loxochelis em Ubatuba. Este padrão pode caracterizar uma estratégia adaptativa para reduzir a competição das larvas de L. loxochelis com a de outras espécies que ocorrem na região. O recrutamento dos juvenis ocorreu principalmente nos meses de inverno em Caraguatatuba e distribuído nos meses de verão e inverno em Ubatuba.

Segundo Fotheringham \& Bagnall (1976), com base em estudo realizado no Texas (EUA), o fato de o assentamento larval ocorrer durante o inverno pode ser vantajoso, pois nesse período há uma grande deposição de pequenas conchas de gastrópodes. De acordo com os mesmos, a estação reprodutiva no inverno pode ser compensadora pela redução na porcentagem de predadores e/ou competidores; além disso, a diversidade e abundância do zooplâncton e de certos predadores foi menor no inverno do que no verão.

Para algumas espécies que ocorrem na costa brasileira, já foram registrados ciclos reprodutivos contínuos com pico de fêmeas ovígeras no verão, como em Clibanarius antillensis (TurRA \& LeIte, 1999), Paguristes tortugae (Mantelatto \& Sousa, 2000) e Paguristes erythrops (GARCIA \& MantelatTo, 2001). Também há registros de ciclos descontínuos: e.g. Calcinus tibicen (Fransozo \& Mantelatto, 1998) e Petrochirus diogenes (BERTINI \& FRANSOZO, 2000), também com pico reprodutivo no verão. Esta variabilidade no perfil reprodutivo é indicativa de uma ampla plasticidade da espécie em adaptar-se às condições ambientais e à coexistência com outras espécies, evitando competição e conseqüente perda de benefícios para os envolvidos, particularmente em L. loxochelis, que apresenta preferência por águas frias e tem o litoral paulista como real limite norte de distribuição e ocorrência em abundância (MARTINELli et al., 2002).

A distribuição de freqüência foi unimodal para ambos os sexos, não ocorrendo variação sazonal da mesma. Mantelatto \& Sousa (2000) registraram distribuição bimodal para machos e para fêmeas ovígeras de Paguristes tortugae, enquanto que para as fêmeas não-ovígeras a distribuição foi unimodal. Bimodalidade e/ou polimodalidade geralmente refletem pulsos de recrutamento, taxas diferenciais de mortalidade entre os sexos e/ou diferenças comportamentais (hábito críptico, migração). A unimodalidade de distribuição é referida como o modelo mais comum em crustáceos decápodes, usualmente refletindo um recrutamento contínuo sem interrupção de classes e taxas de mortalidade constantes (DíAz \& Conde, 1989). Loxopagurus loxochelis seguiu o padrão unimodal, o mais comumente encontrado para anomuros e braquiúros de áreas tropicais.

De acordo com WenNer (1972), em muitos crustáceos marinhos maduros a razão sexual de 1:1 não ocorre. A razão sexual em L. loxochelis diferiu significativamente do teórico esperado de FISHER (1930) (1:1) e foi favorável aos machos nas duas áreas estudadas. O mesmo resultado foi obtido por MARTINELLi et al. (2002) e BERTINi et al. (2004a) com L. loxochelis na região de Ubatuba e também observado por BERTINI \& FRANSOzO (2000) no estudo com Petrochirus diogenes na mesma área. Esse perfil diferiu da maioria dos ermitões estudados em Ubatuba, onde a razão sexual foi favorável às fêmeas (Negreiros-Fransozo \& Fransozo, 1992; Reigada \& SAnTOS, 1997; Fransozo \& Mantelatto, 1998; TuRRA \& Leite, 1999; MANTElatTo \& SousA, 2000). A razão sexual favorável aos machos deve ser em função de uma sobrevivência diferencial entre os sexos após o estágio de megalopa (glaucothöe), ou mesmo a diferentes expectativas de vida entre os sexos (machos com longevidade maior). Esta pode ainda ser uma estratégia reprodutiva, onde uma fêmea possa copular com mais de um macho durante o processo reprodutivo.

Outros fatores podem atuar nesse perfil, como por exemplo, maior gasto de energia voltada para a reprodução, uma longa exposição a predadores e ao estresse físico, diferenças na ecologia comportamental, ou migração de fêmeas maduras para outros habitats, para desovar (GHerardi, 1991). Segundo MANTElatto (2000), fêmeas ovígeras de Callinectes ornatus Ordway, 1863 na enseada de Ubatuba, apresentaram segregação espacial visando proteção à prole e melhor eficiência para a eclosão das larvas.

Quando analisada por classe de tamanho, tanto em Caraguatatuba como em Ubatuba, a razão sexual diferenciou do esperado (1:1), sendo que nas classes intermediárias foi próxima do 1:1 ou favorável às fêmeas e a favor dos machos nas maiores classes de tamanho, como já observado em outros crustáceos (e.g. ABRAMS, 1988; LOWERY \& NELSON, 1988; REIGADA \& SANTOS, 1997; Turra \& Leite, 1999; Garcia \& Mantelatto, 2001; Martinelli et al., 2002). O padrão encontrado em $L$. 
loxochelis pode ser caracterizando como um padrão anômalo (WENNER, 1972). Verificou-se ainda que em Caraguatatuba houve uma estabilização da "curva de Wenner", atingindo $100 \%$ de machos nas duas últimas classes de tamanho. Em Ubatuba só foi registrado 100\% de machos na última classe.

Conforme a hipótese levantada, as duas populações estudadas neste trabalho apresentam dinâmicas de desenvolvimento semelhantes. Com respeito ao maior número de indivíduos coletados em Caraguatatuba em relação à Ubatuba, este deve estar relacionado a características ambientais particulares das duas enseadas, sendo que as duas populações apresentaram indivíduos com tamanhos semelhantes e mesmo padrão de distribuição em classes de tamanho, além de uma alta incidência de machos encontrados em ambas as regiões. Todas essas características estão em concordância com aqueles estudos que avaliaram populações de $L$. loxochelis em áreas mais rasas (BERTINI et al., 2004a; Martinelli et al., 2002). Porém, apenas pesquisas comparativas com espécimes de regiões mais ao sul do Atlântico (Rio Grande do Sul, Argentina) auxiliariam no traçado de um perfil unificado para a espécie.

Agradecimentos. Este trabalho é parte da Dissertação de Mestrado de LAP, realizada com suporte da CAPES. O trabalho de campo teve apoio financeiro da FAPESP - Programa Biota (Proc. 98/07090-3). FLM recebe apoio do Conselho de Desenvolvimento Científico e Tecnológico (CNPq-PQ). Agradecimentos especiais são devotados para Dr. Adilson Fransozo (UNESP), pelo suporte e facilidades durante o trabalho de campo; para os colegas do NEBECC e membros do Laboratório de Bioecologia e Sistemática de Crustáceos da FFCLRP/USP pela ajuda durante o trabalho de campo; para o Programa de PG em Ciências, Área: Biologia Comparada da FFCLRP/USP, pelo suporte nas coletas; para Marcelo Scelzo (Universidade de Mar del Plata) e Roberto Shimizu (IB/USP) e assessores ad hoc, pelos comentários e sugestões. Todos os procedimentos realizados neste estudo estão de acordo com as leis estaduais e federais.

\section{REFERÊNCIAS BIBLIOGRÁFICAS}

Abrams, P. A. 1988. Sexual difference in resource use in hermit crabs; consequences and causes. In: ChelazzI, G. \& VANNINI, M. eds. Behavioral adaptation to intertidal life. New York, Plenum. Pp. 283-296

Asakura, A. 1987. Population ecology of the sand-dwelling hermit crab Diogenes nitidimanus Terao: 3. Mating System. Bulletin of Marine Science 41(2):282-288.

Asakura, A. \& Kikuchi, T. 1984. Population ecology of the sand dwelling hermit crab, Diogenes nitidimanus. Terao. 2. Migration and life history. Publications from the Amakusa Marine Biological Laboratory 7(2):109-123.

Barros, C. E.; Corres, I. C. S.; Baitelli, R. \& Elias, A. R. D. 1997. Aspectos sedimentares da enseada de Caraguatatuba, litoral do Estado de São Paulo. Anais da Academia Brasileira de Ciências 69(1):19-36.

Bertini, G. \& Fransozo, A. 2000. Population dynamics of Petrochirus diogenes (Crustacea, Anomura, Diogenidae) in Ubatuba Region, São Paulo, Brazil. Crustacean Issues 12:331-342.

2004. Bathymetric distribution of brachyuran crabs (Crustacea, Decapoda) communities on coastal soft bottoms off southeastern Brazil. Marine Ecology Progress Series 279: 193-200.

Bertini, G.; Fransozo, A. \& Braga, A. A. 2004a. Ecological distribution and reproductive period of the hermit crab Loxopagurus loxochelis (Anomura, Diogenidae) on the northern coast of São Paulo State, Brazil. Journal of Natural History 38(18):2331-2344.
Bertini, G.; Fransozo, A. \& Melo, G. A. S. 2004b. Biodiversity of brachyuran crabs (Crustacea: Decapoda) from nonconsolidated sublittoral bottom on the northern coast of São Paulo State, Brazil. Biodiversity and Conservation 13:2185-2207

Biagi, R.; Meireles, A. L.; Scelzo, M. A. \& Mantelatto, F. L. 2006. Comparative study of shell choice by the southern endemic hermit crab Loxopagurus loxochelis from Brazil and Argertina. Revista Chilena de Historia Natural 79(4):481-487.

Castro-Filho, B. M.; Miranda, L. B. \& Miyao, S. Y. 1987. Condições hidrográficas na plataforma continental ao largo de Ubatuba: variações sazonais e em média escala. Boletim do Instituto Oceanográfico 35(2):135-151.

Costa, R. C.; Fransozo, A.; Mantelatto, F. L. \& Castro, R. H. 2000. Occurrence of shrimp species (Crustacea: Decapoda: Natantia: Penaeidea and Caridea) in Ubatuba Bay, Ubatuba, SP, Brazil. Proceedings of Biological Society of Washington 113(3):776-781.

Díaz, H. \& Conde, J. E. 1989. Population dynamics and life history of the mangrove crab Aratus pisonii (Brachyura, Grapsidae) in the marine environment. Bulletin of Marine Science 45(1): 148-163.

FISHER, R. A. 1930. The genetical theory of natural selection. 2 ed. New York, Doven. 291p.

Fotheringham, N. \& Bagnall, R. A. 1976. Seasonal variation in the occurrence of planktonic larvae of sympatric hermit crabs. Journal of Experimental Marine Biology and Ecology 21:279-287

Fransozo, A. \& Mantelatto, F. L. 1998. Population structure and reproductive period of the hermit crab Calcinus tibicen (Decapoda: Diogenidae) in the region of Ubatuba, São Paulo, Brazil. Journal of Crustacean Biology 18(4):738-745.

Fransozo, A.; Mantelatto, F. L.; Bertini, G.; Fernandez-Góes, L. C. \& Martinelli, J. M. 1998. Distribution and assemblages of anomuran crustaceans in Ubatuba Bay, north coast of São Paulo State, Brazil. Acta Biologica Venezuelica 18(4):17-25.

Garcia, R. B. \& Mantelatto, F. L. 2001. Population dynamics of the hermit crab Paguristes erythrops (Diogenidae) from Anchieta Island, southern Brazil. Journal of the Marine Biological Association of the United Kingdom 81:955-960.

2006. Relative growth and sexual maturity of the hermit crab Paguristes erythrops (Anomura, Diogenidae) from South Atlantic. Hydrobiologia 559:247-254.

GHerardi, F. 1991. Relative growth, population structure, and shell-utilization of the hermit crab Clibanarius erythropus in the Mediterranean. Oebalia 17:181-196.

IngLe, R. 1993. The hermit crabs of the northeastern Atlantic Ocean and Mediterranean Sea - an illustrated key. London, Chapman \& Hall. 495p.

LANCASTER, I. 1988. Pagurus bernhardus (L.) - an introduction to the natural history of hermit crabs. Field Studies 7:189-238.

Lowery, W. A. \& Nelson, W. G. 1988. Population ecology of the hermit crab Clibanarius vittatus (Decapoda: Diogenidae) at Sebastian Inlet, Florida. Journal of Crustacean Biology 8(4):548-556

Mantelatto, F. L. 2000. Allocation of the portunid crab Callinectes ornatus (Decapoda: Brachyura) in the Ubatuba Bay, northern coast of São Paulo State, Brazil. Crustacean Issues 12(2):431-443.

Mantelatto, F. L. \& Fransozo, A. 1999. Characterization of the physical and chemical parameters of Ubatuba Bay, northern coast of São Paulo State, Brazil. Revista Brasileira de Biologia 59(1):23-31.

Mantelatto, F. L. \& Garcia, R. B. 2002. Hermit crab fauna from the infralitoral area of Anchieta Island (Ubatuba, Brazil). In: Briones, E. E. \& Alvarez, F. eds. Modern Approaches to the Studies of Crustacean. New York, Kluwer. Pp. 137-144.

Mantelatto, F. L. \& Martinelli, J. M. 2001. Relative growth and sexual dimorphism of the South Atlantic hermit crab Loxopagurus loxochelis (Anomura, Diogenidae) from Ubatuba, Brazil. Journal of Natural History 35(3):429-437.

Mantelatto, F. L. \& Meireles, A. L. 2004. The importance of shell occupation and shell availability in the hermit crab Pagurus brevidactylus (Stimpson, 1859) (Paguridae) population from Southern Atlantic. Bulletin of Marine Science 75(1):27-35. 
Mantelatto, F. L. \& Sousa, L. M. 2000. Population biology of the hermit crab Paguristes tortugae Schmitt, 1933 (Anomura, Diogenidae) from Anchieta Island, Ubatuba, Brazil. Nauplius 8(2):185-193.

Mantelatto, F. L.; Christofoletti, R. A. \& Valenti, W. C. 2005. Population structure and growth of the hermit crab Pagurus brevidactylus (Anomura: Paguridae) from the northern coast of São Paulo, Brazil. Journal of the Marine Biological Association of the United Kingdom 85(1):127-128.

Mantelatto, F. L.; Martinelli, J. M. \& Fransozo, A. 2004. Temporal - spatial distribution of the hermit crab Loxopagurus loxochelis (Decapoda, Anomura, Diogenidae) from Ubatuba Bay, São Paulo State, Brazil. Revista de Biología Tropical 52(1):47-55

Mantelatto, F. L.; Garcia, R. B.; Martinelli, J. M. \& Hebling, N. J. 2001. On a record of Dardanus venosus (H. Milne Edwards) (Crustacea, Anomura) from the São Paulo State, Brazil. Revista Brasileira de Zoologia 18(1):71-73.

Mantelatto, F. L.; Robles, R.; Biagi, R. \& Felder, D. L. 2006. Molecular analysis of the taxonomic and distributional status based for the hermit crab genera Loxopagurus Forest, 1964, and Isocheles Stimpson, 1858 (Decapoda, Anomura, Diogenidae). Zoosystema 28(2):495-506.

Martinelli, J. M. \& Mantelatto, F. L. 1998. Occurrence of exobionts in gastropod shells occupied by the hermit crab Loxopagurus loxochelis (Anomura: Diogenidae) in Ubatuba Bay (SP) Brazil. In: Anais do IV Simpósio de Ecossistemas Brasileiros 2(104):221-226. São Paulo, Publicações ACIESP.

1999. Shell utilization by the hermit crab Loxopagurus loxochelis (Diogenidae) in Ubatuba Bay, Brazil. In: Schram, F. R. \& Vanpel Klein, J. C. eds. Crustaceans and the Biodiversity Crisis 1:719-731.

Martinelli, J. M.; Mantelatto, F. L. \& Fransozo, A. 2002. Population structure and breeding season of the South Atlantic hermit crab, Loxopagurus loxochelis (Anomura, Diogenidae) from the Ubatuba region, Brazil. Crustaceana 75(6):791-802.

McLaughlin, P. A. 2003. Illustrated keys to families and genera of the superfamily Paguroidea (Crustacea: Decapoda: Anomura), with diagnoses of genera of Paguridae. In: Lemaitre, R. \& Tudge, C. C. eds. Biology of the Anomura. Proceedings of a symposium at the Fifth International Crustacean Congress, Melbourne, Australia, 9 - 13 July 2001. Memoirs of the Museum of Victoria 60(1):111-144

Meireles, A. L. \& Mantelatto, F. L. 2005. Shell use by the Pagurus brevidactylus (Anomura, Paguridae): a comparison between laboratory and field conditions. Acta Zoologica Sinica 51(5):813-820.

Meireles, A. L.; Terossi, M.; Biagi, R. \& Mantelatto, F. L. 2006. Spatial and seasonal distribution of the hermit crab Pagurus exilis (Benedict, 1892) (Decapoda: Paguridae) in the Southwestern coast of Brazil. Revista de Biología Marina y Oceanografía 14(1):87-95.

Meireles, A. L.; Biagi, R.; Fransozo, A. \& Mantelatto F. L. (no prelo). Os Ermitões (Crustacea, Anomura). In: Amaral, A. C. Z. \& Nallin, S. A. H. orgs. Biodiversidade e ecossistemas bentônicos marinhos do Litoral Norte de São Paulo sudeste do Brasil. Editora UNICAMP, Campinas. p.1-10.
Melo, G. A. S. 1999. Manual de identificação dos Crustacea Decapoda do litoral brasileiro: Anomura, Thalassinidea, Palinuridea e Astacidea. São Paulo, Plêiade. 551p.

Negreiros-Fransozo, M. L. \& Fransozo, A. 1992. Estrutura populacional e relação com a concha em Paguristes tortugae Schmitt, 1933 (Decapoda, Diogenidae), no litoral norte do Estado de São Paulo, Brasil. Naturalia 17(1):31-42.

Negreiros-Fransozo, M. L.; Fransozo, A. \& Hebling, N. J. 1991. Estrutura populacional e determinação do tamanho da concha em quatro espécies de ermitões (Crustacea, Decapoda, Anomura) do litoral paulista. Biotemas 4(2):135-148.

Nucci, P. R. \& Hebling, N. J. 2004. A new species of Paguristes (Decapoda: Paguroidea: Diogenidae) from Brazil. Nauplius 12(2):66-70.

Nucci, P. R. \& Melo, G. A. S. 2003. A new species of Pagurus (Decapoda: Anomura: Paguridae) from Brazil. Journal of the Marine Biological Association of the United Kingdom 83(2):351-353.

Pires-Vanin, A. M. S.; Rossi-Wongtschowski, C. L. Del B.; Aidar, E.; Mesquita, H. De S. L.; Soares, L. S. H.; Katsuragawa, M. \& Matsuura, I. 1993. Estrutura e função do ecossistema de plataforma continental do Atlântico Sul brasileiro: síntese dos resultados. Publicação Especial do Instituto Oceanográfico (10):217-231.

Reigada, A. L. D. \& Santos, S. 1997. Biologia e relação com a concha em Clibanarius vittatus (Bosc, 1802) (Crustacea, Diogenidae) em São Vicente, SP, Brasil. Brazilian Archives of Biology and Technology 40(4):941-952.

Rieger, P. J. \& D'Incao, F. 1991. Distribuição de larvas de Loxopagurus loxochelis (Decapoda, Diogenidae) na região adjacente à Barra de Rio Grande, RS. Nerítica 6(1-2):93-106.

Scelzo, M. A. 1976. Larvas de los crustáceos decápodos anomuros identificados en las aguas marinas Argentinas. Physis 35(90):37-45.

Scelzo, M. A. \& Boschi, F. F. 1973. Aportes al conocimiento de la distribución geográfica de los crustáceos decápodos anomura del Atlántico Sudoccidental, frente a las Costas Argentinas. Trabalhos do $V$ Congresso Latino Americano de Zoologia 1:204-216.

Scelzo, M. A.; Mantelatto, F. L. \& Tudge C. 2004. Spermatophore morphology of the hermit crab Loxopagurus loxochelis (Moreira, 1901) (Anomura, Diogenidae) from the southwestern Atlantic. Invertebrate Reproduction and Development 46(1):1-9.

Torati, L. S. \& Mantelatto F. L. 2008. Uncommon mechanism of egg incubation in the endemic Southern hermit crab Loxopagurus loxochelis: how is this phenomenon related to egg production? Acta Zoologica 89(1):79-85.

Turra, A. \& Leite F. P. P. 1999. Population structure and fecundity of the hermit crab Clibanarius antillensis Stimpson 1862 (Anomura, Diogenidae) in southeastern Brazil. Bulletin of Marine Science 64(2):281-289.

Wenner, A. M. 1972. Sex ratio as a function of size in marine Crustacea. The American Naturalist 106:321-350.

ZAR, J. H. 1996. Biostatistical Analysis. New Jersey, PrenticeHall. $907 \mathrm{p}$

Recebido em outubro de 2006. Aceito em julho de 2007. ISSN 0073-4721

Artigo disponível em: www.scielo.br/isz 